\title{
Risk Factors, Clinical Characteristics, and Treatment Differences Between Residents With and Without Nursing Home- and Non-Nursing Home-Acquired Clostridium difficile Infection
}

\author{
Barbara J. Zarowitz, PharmD; Carrie Allen, PharmD; \\ Terrence O'Shea, PharmD; and Marcie E. Strauss, MPH
}

\begin{abstract}
BACKGROUND: The incidence of Clostridium difficile infection (CDI) in nursing home residents is believed to be high because of the prevalence of predisposing factors such as decreased immune response, multiple comorbidities, medications, increased risk of infection, close proximity of residents, and recent hospitalization. Yet, specific information on CDI in this population is scarce.

OBJECTIVES: To investigate differences in clinical and demographic characteristics, treatment, and underlying comorbidities in residents who acquired CDI preadmission (non-nursing home-acquired [NNH-Acquired]) compared with those who acquired CDI after admission to a nursing home (nursing home-acquired [NH-Acquired]) and matched controls.

METHODS: We conducted a retrospective case-control study of CDI in nursing home residents with a cross-sectional and longitudinal aspect of linked and de-identified pharmacy claims and Minimum Data Set data (MDS) 2.0 records from 0ctober 1, 2009, to September 30, 2010. The control group was frequency matched 1:1 for gender, race, and age range to residents with CDI.
\end{abstract}

RESULTS: Of 195,498 residents, 5,044 (2.6\%) had a diagnosis of CDI. Compared with controls, CDI patients had less severe cognitive impairment $(P<0.01)$ and more severe functional impairment $(P<0.01)$, incontinence $(P<0.01)$, and diarrhea $(P<0.01)$. They were more likely to (a) have diabetes, stroke, heart failure, cancer, renal failure, and infections; (b) be treated with antibiotics, corticosteroids, megestrol, and proton pump inhibitors; and (c) be discharged to the hospital $(29.3 \%$ vs. $14.7 \%, P=0.001)$ than controls. NNH-Acquired CDI was 3 times more prevalent than $\mathrm{NH}$-Acquired CDI. Most residents with NNH-Acquired CDI (85.0\%) came from acute care hospitals and were more likely to have heart disease, cancer, and infections, while those with $\mathrm{NH}$-Acquired CDI tended to have more cognitive impairment, reliance on staff for activities of daily living, incontinence, and stroke. Thirty-day retreatment rates for $\mathrm{NH}-\mathrm{Acquired} \mathrm{CDI}$ and NNHAcquired CDI with metronidazole were $72.7 \%$ and $68.4 \%$, and with vancomycin were $83.9 \%$ and $69.3 \%$, respectively. The facility (Medicare Part A) was the payer for $93.6 \%$ of NNH-Acquired CDI and $75 \%$ of $\mathrm{NH}-$ Acquired CDI treatment; Medicare Part D was the prevalent secondary payer for NNHAquired CDI (19.4\%) and NH-Acquired CDI (37.5\%).

CONCLUSIONS: Residents with CDI had more comorbidities, and the NNHAcquired group bore a higher burden of illness, resulting in differing treatment patterns and outcomes than the NH-Acquired CDI group.

J Manag Care Spec Pharm. 2015;21(7):585-95

Copyright $\odot 2015$, Academy of Managed Care Pharmacy. All rights reserved.

\section{What is already known about this subject}

Nursing home residents are predisposed to Clostridium difficile infection (CDI) due to a decreased immune response, comorbidities, medications, and an increased risk of infection.

The incidence of CDI in long-term care facilities may be as high as 3.72 cases per 1,000 resident days, and CDI carries an increased burden of illness for the nursing home residents and care staff.

As many as $67 \%$ of nursing home CDI cases may be attributable to a recent hospital discharge.

\section{What this study adds}

Nursing home residents are 3 times more likely to acquire CDI before entering the nursing home than after nursing home admission, and $85 \%$ of the time, residents have been admitted to the nursing home from an acute care setting.

Initial treatment and retreatment with vancomyin oral solution was more common in NNH-Acquired CDI, perhaps because of more severe illness, whereas initial treatment and retreatment with metronidazole was more prevalent in nursing homeacquired (NH-Acquired) CDI.

There is a substantial burden of illness for nursing home residents with CDI, and residents with NNH-Acquired CDI are more likely to have severe underlying illness and be readmitted to the hospital compared with patients with NH-Acquired CDI.

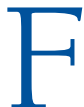
actors that may predispose nursing home residents to Clostridium difficile infection (CDI) include a decreased immune response, multiple comorbidities, medications, and an increased risk of infection. ${ }^{1-6}$ CDI and C. difficileassociated diarrhea (CDAD) are problematic in these facilities because of the close proximity of residents, challenges with effective isolation of infected individuals, and difficulty eradicating $C$. difficile spores in this environment. ${ }^{7}$ Because many residents are admitted to nursing homes following an acute hospital stay, the recent finding that $67 \%$ of nursing homeonset CDI/CDAD cases occur in patients recently discharged from an acute care hospital is of concern. ${ }^{8}$ Further, when nursing home residents acquire CDI in the nursing home and not from an acute care hospital, the underlying risk factors, burden of illness, and disposition may be different. ${ }^{9}$ The incidence of 
CDI in long-term care facilities may be as high as 3.72 cases per 1,000 resident days, although it is not known what proportion is due to internal versus external sources of infection. ${ }^{7}$

Our objectives for this study were to describe demographic and clinical characteristics, physical and cognitive function, and pharmacologic treatment of residents with a diagnosis of CDI in U.S. nursing homes. We identified differences in comorbidities (e.g., renal disease and cancer); cognition; functional status; hospitalizations; and mortality between age- and gender-matched residents without CDI. Additionally, we identified clinical parameters that were frequently associated with CDI in residents who acquired CDI prior to their nursing home admission (non-nursing home-acquired [NNH-Acquired]) compared with those acquiring CDI after admission (nursing homeacquired [NH-Acquired]).

\section{Methods}

After obtaining an exemption of institutional review board review and waiver of authorization from Sterling Institutional Review Board, data extracts of prescription claims and Minimum Data Set (MDS) version 2.0 assessments for residents in Omnicare-serviced nursing homes for the incident time period were obtained from the Omnicare Senior Health Outcomes data repository. These data are from about 200 pharmacies serving more than 1.4 million residents in about 19,000 long-term care facilities in 48 states (excluding Hawaii and Alaska). Data extracts were made HIPAA-compliant by de-identification at the resident, prescriber, facility, and pharmacy level. The study period was characterized using a data extract of linked MDS 2.0 and prescription claims data from October 1, 2009, through September 30, 2010. CDI was identified in unique residents who had either field I2b completed (specific to documenting CDI) or an International Classification of Diseases, Ninth Revision, Clinical Modification (ICD-9-CM) diagnosis of $C$. difficile infection (008.45) and treatment with either oral vancomycin or metronidazole.

For the purpose of identifying NNH-Acquired CDI, all recorded diagnoses of CDI that first appeared in a New Admission MDS Assessment, Medicare Readmission/Return MDS Assessment, Medicare 5-Day Assessment, or Medicare 14-Day Assessment with prescription claims data were considered to be NNH-Acquired. The first appearance of the diagnosis of CDI on any other type of MDS assessment was considered to be NH-Acquired.

Each resident was assigned a unique identification key to permit longitudinal tracking at the prescription claims level. The unique identification key does not change over time and allows the resident to be tracked despite new facility identification assignments due to changes in facility ownership or reconfiguration of pharmacies subsequent to a merger. Diagnostic, prescription claims, and MDS data from 2 large nursing home chains-SAVA Senior Care and HCR Manor Care facilitieswere excluded due to pre-existing contractual agreements.
Evaluation of cognitive function was based on MDS 2.0 fields B1-Comatose, B2a-Short Term Memory, B4-Cognitive Skills for Daily Decision Making, C4-Making Self Understood, and GlhA-Eating Self-Performance. From these parameters, a Cognitive Performance Scale (CPS) was calculated wherein a score of 0 indicated no cognitive impairment and a score of 6 indicated very severe impairment. For the purposes of this analysis, moderate-to-severe cognitive impairment was defined as CPS scores of 3-6. ${ }^{10}$

The CDI incidence rate per 10,000 resident days was calculated as the number of CDI incidents per month per number of resident days $\times 10,000$. The calculation excludes recurrent events. Figure 1 summarizes the patient selection flow for this study.

\section{Data Analysis}

The control group was frequency matched for age, race, and gender. A descriptive univariate analysis was performed for baseline characteristics between cases and controls.

Comparison of differences between NH-Acquired and NNH-Acquired CDI in underlying diagnoses, cognition, function, renal disease, immobility, hospitalizations, and risk factors that may predispose residents to contract CDI were assessed by a chi-squared test for binary variables, Student's t-tests for continuous variables, and Wilcoxon 2-sample tests for ordinal variables (e.g., activities of daily living [ADL] scores). Comparisons in which data were more than $20 \%$ sparse, defined as having a count less than 5 , were calculated using exact $P$ values using Fisher's exact test.

During the study period, many residents had more than 1 MDS assessment completed. When this occurred, the assessment that recorded the more severe condition was used.

\section{Results}

Of 195,498 unique residents with MDS data, 5,044 (2.6\%) had CDI, which corresponded to an estimated CDI prevalence of 0.979 per 10,000 resident days. Among the 2,048 unique residents with CDI, an admission MDS, and prescription claims data, 1,609 (78.6\%) were NNH-Acquired, and 439 (21.4\%) were $\mathrm{NH}$-Acquired. The vast majority (85\%) of NNH-Acquired CDI admissions were from acute care hospitals, with fewer originating from other nursing homes (2.9\%), the community (1.8\%), rehabilitation hospitals (1.0\%), other settings $(0.5 \%)$, or unknown admission location (8.8\%).

Using a matched control group of residents who did not have a diagnosis of CDI, we found that residents with CDI had more severe functional impairment, more urinary and bowel incontinence, and more diarrhea (see Appendix, available in online article). However, they had less severe cognitive impairment. Residents with CDI were more likely to have diabetes, stroke, congestive heart failure, cardiac arrhythmias, renal failure, cancer, urinary tract infection, pneumonia, antibiotic-resistant infection, and foot infection (Figure 2). These characteristics are consistent with a larger number of underlying comorbidities in the CDI group. Similarly, residents 


\section{FIGURE 1 Patient Selection}

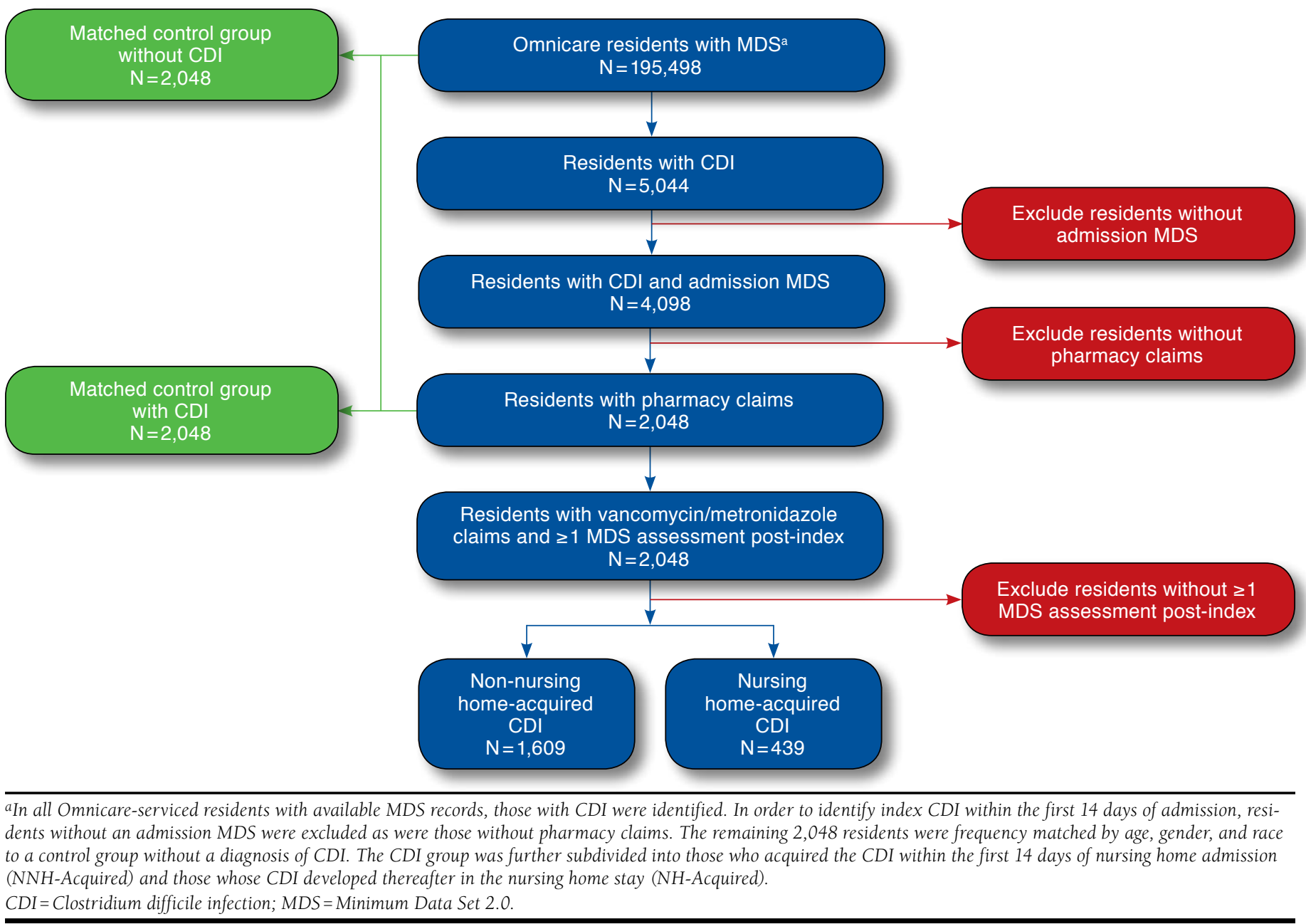

with CDI used more cephalosporins, penicillins, carbapenems, monopenems, clindamycin, aminoglycosides, tigecycline, corticosteroids, megestrol, and proton pump inhibitors than the matched control residents. The residents with CDI were more likely to be undergoing dialysis (6.2\% vs. $3.1 \%)$ and be discharged to the hospital (29.3\% vs. $14.7 \%)$. We were unable to evaluate why the residents were readmitted to the hospital, given that the trial was not designed to evaluate hospital readmission. Nevertheless, the higher hospitalization rate in CDI residents supports our findings that these residents were sicker with greater prevalence of chronic diseases and infections.

Table 1 depicts selected comparative demographic, functional, and cognitive characteristics as well as comorbidities and medication treatment of residents with $\mathrm{NH}$-Acquired versus NNH-Acquired CDI. Residents with NH-Acquired CDI were more likely to be cognitively impaired; be more functionally dependent on staff for ADLs; and have more urinary incontinence, bowel incontinence, and diarrhea than residents with NNH-Acquired CDI. Additionally, residents with $\mathrm{NH}$-Acquired CDI were less likely to have congestive heart failure, cardiac arrhythmias, renal failure, cancer, antibioticresistant infection, septicemia, and wound infection than those who acquired CDI prior to nursing home admission. However, residents with NH-Acquired CDI were more likely to have had a cerebrovascular accident and falls.

Use of medications coincident with CDI were similar between the NH-Acquired and NNH-Acquired CDI groups with the exception of number of antibiotics, including fluoroquinolones, cephalosporins, penicillins, sulfamethoxazole-trimethoprim, nitrofurantoin, macrolides, doxycycline, clindamycin, linezolid, aminoglycosides, and tigecycline, all of which were used by a higher percentage of residents with $\mathrm{NH}$-Acquired CDI than NNH-Acquired CDI while in the nursing home. Residents with NH-Acquired CDI were more likely 
Risk Factors, Clinical Characteristics, and Treatment Differences Between Residents With and Without Nursing Home- and Non-Nursing Home-Acquired Clostridium difficile Infection

FIGURE 2 Comparison of Clinical Characteristics, Underlying Comorbidities, Medication Use, and Outcomes in Residents with CDI Versus Matched Control Residents Without CDI

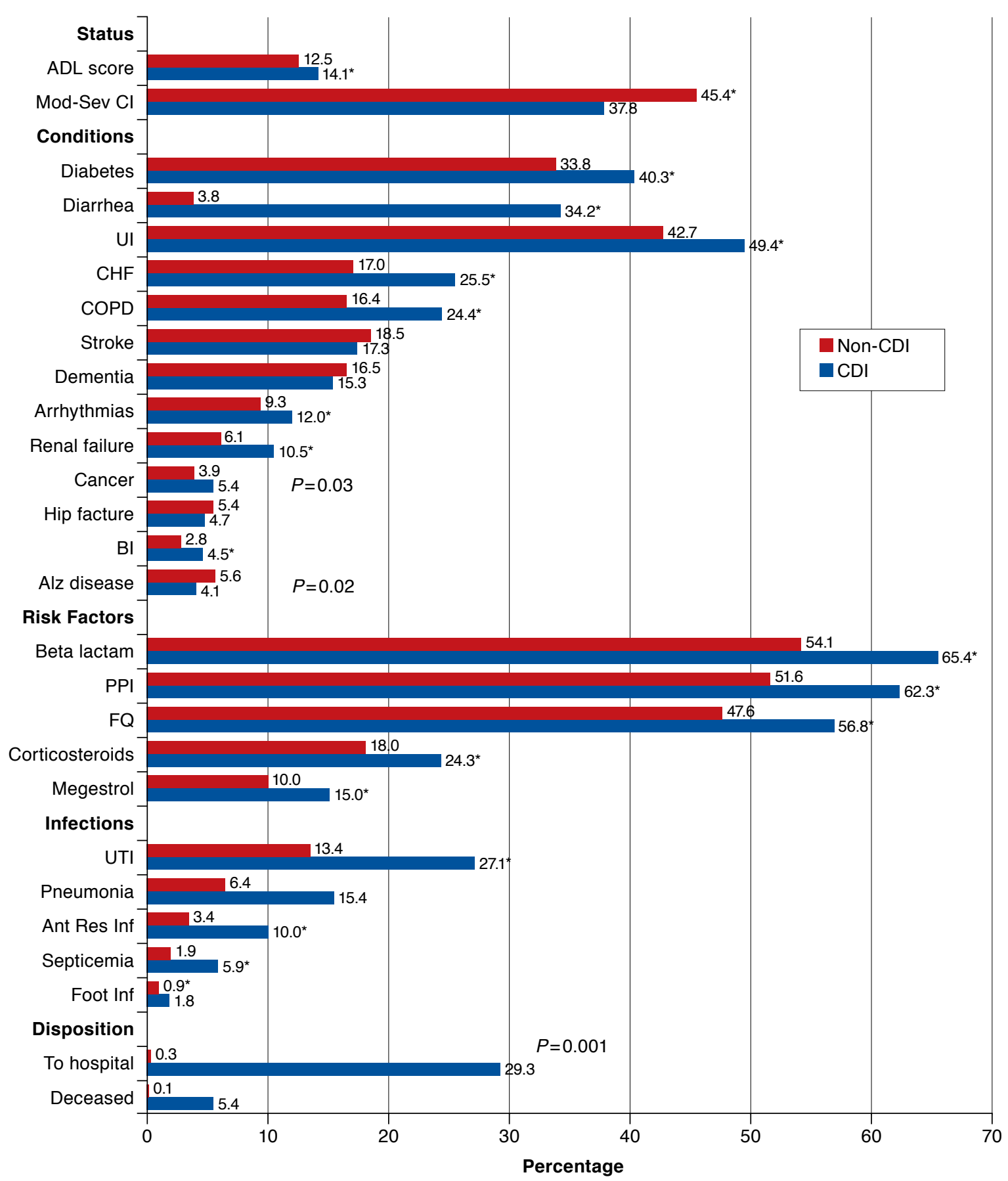

Note: Parameters depicted with * were statistically significantly different at $P<0.01$.

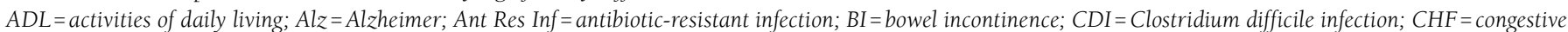
heart failure; $\mathrm{COPD}=$ chronic obstructive pulmonary disease; $F Q=$ fluoroquinolone; Inf=infection; Mod-Sev CI= moderate-to-severe cognitive impairment; $P P I=$ proton pump inhibitor; UI= urinary incontinence; UTI= urinary tract infection. 


\section{Risk Factors, Clinical Characteristics, and Treatment Differences Between Residents With and Without Nursing Home- and Non-Nursing Home-Acquired Clostridium difficile Infection}

TABLE 1 Comparative Demographic, Functional, and Cognitive Characteristics; Comorbidities; and Treatment of Residents with $\mathrm{NH}$-Acquired Versus NNH-Acquired CDI

\begin{tabular}{|c|c|c|c|c|}
\hline \multirow{2}{*}{$\begin{array}{l}\text { MDS Data Element } \\
\text { Number of unique residents with CDI }\end{array}$} & $\begin{array}{l}\text { Total } \\
\text { n (\%) }\end{array}$ & $\begin{array}{l}\text { NNH-Acquired } \\
\text { n (\%) }\end{array}$ & $\begin{array}{c}\text { NH-Acquired } \\
\text { n (\%) }\end{array}$ & \multirow{2}{*}{$\frac{P \text { Value }}{\text { N/A }}$} \\
\hline & $2,048 \quad(100.0)$ & $(78.6)$ & $(21.4)$ & \\
\hline \multicolumn{5}{|l|}{ Demographics } \\
\hline Female & 1,257 & $(60.5)$ & $(64.5)$ & 0.134 \\
\hline Age, mean \pm SD & $78.3 \pm 12.2$ & $78.1 \pm 12.2$ & $79.0 \pm 12.4$ & 0.335 \\
\hline Race, white & 1,576 & $(76.6)$ & $(78.4)$ & 0.979 \\
\hline \multicolumn{5}{|l|}{ Cognitive and functional status } \\
\hline Moderate-to-severe cognitive impairment & $(37.8)$ & $(35.5)$ & $(46.2)$ & 0.001 \\
\hline Composite ADL score, mean \pm SD & $14.1 \pm 3.2$ & $14.0 \pm 3.0$ & $14.4 \pm 3.2$ & 0.006 \\
\hline \multicolumn{5}{|l|}{ Co-existing conditions } \\
\hline Urine incontinence & 1,010 & $(46.8)$ & $(58.3)$ & 0.001 \\
\hline Bowel incontinence & 1,219 & $(56.9)$ & $(69.0)$ & 0.001 \\
\hline Diarrhea & $(34.2)$ & $(33.1)$ & $(38.3)$ & 0.047 \\
\hline Diabetes & $(40.5)$ & $(41.2)$ & $(36.7)$ & 0.064 \\
\hline $\mathrm{CHF}$ & $(25.5)$ & $(26.5)$ & $(21.9)$ & 0.047 \\
\hline COPD & $(24.4)$ & $(25.1)$ & $(21.6)$ & 0.134 \\
\hline CVA & $(17.3)$ & $(15.2)$ & $(25.1)$ & 0.001 \\
\hline Dementia, non-AD & $(15.3)$ & $(15.4)$ & $(14.8)$ & 0.754 \\
\hline Cardiac arrhythmias & $(12.0)$ & $(13.6)$ & $(6.2)$ & 0.001 \\
\hline Renal failure & $(10.5)$ & $(11.4)$ & $(7.1)$ & 0.009 \\
\hline Cancer & $(5.4)$ & $(5.9)$ & $(3.4)$ & 0.041 \\
\hline $\mathrm{AD}$ & $(4.1)$ & $(3.7)$ & $(5.7)$ & 0.058 \\
\hline \multicolumn{5}{|l|}{ Infections } \\
\hline Urinary tract infection & $(27.1)$ & $(26.5)$ & $(28.9)$ & 0.318 \\
\hline Pneumonia & $(15.4)$ & $(16.5)$ & $(11.6)$ & 0.013 \\
\hline Antibiotic-resistant infection & $(10.0)$ & $(10.6)$ & $(7.7)$ & 0.024 \\
\hline Septicemia & $(5.9)$ & $(6.5)$ & (3.9) & 0.041 \\
\hline Wound infection & $(5.1)$ & $(5.7)$ & $(3.2)$ & 0.038 \\
\hline \multicolumn{5}{|l|}{ Falls and fractures } \\
\hline Falls & $(37.2)$ & $(36.0)$ & $(41.5)$ & 0.035 \\
\hline Fractures & $(10.9)$ & $(11.1)$ & $(10.3)$ & 0.678 \\
\hline Hip fractures & $(4.7)$ & $(4.2)$ & $(6.4)$ & 0.059 \\
\hline
\end{tabular}

$A D=$ Alzheimer's disease; $A D L=$ activities of daily living; $C D I=$ Clostridium difficile infection; $C H F=$ congestive heart failure; $C O P D=$ chronic obstructive pulmonary disease; $C V A=$ cerebrovascular accident; MDS=Minimum Data Set, N/A = not applicable; NH-Acquired=nursing home-acquired; NNH-Acquired =non-nursing homeacquired; $S D=$ standard deviation

to receive nutrients by feeding tube. The NNH-Acquired CDI group was more likely to be undergoing dialysis $(7.0 \%$ vs. $3.4 \%)$ and be discharged to the hospital (32.3\% vs. $18.2 \%)$ than residents who acquired $\mathrm{CDI}$ in the nursing home.

The facility (Medicare Part A) was the most prevalent payer for NNH-Acquired (93.6\%) and NH-Acquired (75.0\%) CDI treatment. Medicare Part D was the payer $37.5 \%$ of the time for NH-Acquired CDI but for only $19.4 \%$ of medication claims for residents with $\mathrm{NNH}$-Acquired $\mathrm{CDI}$.

When we examined the patterns of metronidazole and vancomycin use in residents with initial and subsequent CDI treatment, several key findings emerged (Table 2). NH-Acquired residents were more likely to require retreatment within 30 days than NNH-Acquired residents ( $75 \%$ vs. $69 \%$ ), and the highest rates of within 30-day retreatment were for $\mathrm{NH}$-Acquired residents treated with oral vancomycin solution (84\%). Oral metronidazole was the most common initial treatment of CDI. NH-Acquired CDI residents were more likely to be given a second course of oral metronidazole than $\mathrm{NNH}$-Acquired CDI ( $47.8 \%$ vs. $35.6 \%$, respectively), and approximately $77 \%$ were given a second course within 30 days of initial treatment with metronidazole. Significantly more residents with $\mathrm{NNH}-$ Acquired CDI were treated initially with oral vancomycin and then given a second course of oral vancomycin compared with those with NH-Acquired CDI. Most NNH-Acquired CDI residents receiving a second course of treatment were given oral vancomycin within 30 days of the initial course of therapy. These findings are consistent with current CDI treatment guidelines from the Society of Healthcare Epidemiology of America (SHEA) and the Infectious Disease Society of America (IDSA).11 
TABLE 2 Metronidazole and Vancomycin Use and Time Course in $\mathrm{NNH}$-Acquired CDI Compared with $\mathrm{NH}$-Acquired CDI

\begin{tabular}{|c|c|c|c|c|c|c|c|}
\hline \multirow{2}{*}{$\begin{array}{l}\text { Medication and Time Course } \\
\text { Metronidazole ( } 1 \text { course only, without a vancomycin course) }\end{array}$} & \multicolumn{2}{|c|}{$\begin{array}{l}\text { CDI Total } \\
\text { n (\%) }\end{array}$} & \multicolumn{2}{|c|}{$\begin{array}{l}\text { NNH-Acquired } \\
\text { n (\%) }\end{array}$} & \multicolumn{2}{|c|}{$\begin{array}{l}\text { NH-Acquired } \\
\text { n (\%) }\end{array}$} & \multirow{2}{*}{$\frac{P \text { Value }}{0.001}$} \\
\hline & 627 & $(30.6)$ & 493 & $(30.6)$ & 134 & $(30.5)$ & \\
\hline Metronidazole ( $>1$ course) & 783 & $(38.2)$ & 573 & $(35.6)$ & 210 & $(47.8)$ & \\
\hline Time between courses & & & & & & & 0.990 \\
\hline$<30$ days & 603 & $(77.0)$ & 442 & $(77.1)$ & 161 & $(76.7)$ & \\
\hline 31-90 days & 137 & $(17.5)$ & 100 & $(17.5)$ & 37 & $(17.6)$ & \\
\hline$>90$ days & 43 & $(5.5)$ & 31 & $(5.4)$ & 12 & $(5.7)$ & \\
\hline Vancomycin ( 1 course only, without metronidazole course) & 196 & $(9.6)$ & 177 & $(11.0)$ & 19 & $(4.3)$ & 0.001 \\
\hline Vancomycin (> l course) & 498 & $(24.3)$ & 399 & $(24.8)$ & 99 & $(22.6)$ & \\
\hline Time between courses & & & & & & & 0.416 \\
\hline$<30$ days & 465 & $(93.4)$ & 370 & $(92.7)$ & 95 & $(96.0)$ & \\
\hline 31-90 days & 29 & $(5.8)$ & 25 & $(6.3)$ & 4 & $(4.0)$ & \\
\hline$>90$ days & 4 & $(0.8)$ & 4 & $(1.0)$ & 0 & $(0.0)$ & \\
\hline Metronidazole ( 1 course only) and vancomycin ( 1 course only) & 159 & $(7.8)$ & 130 & $(8.1)$ & 29 & $(6.6)$ & 0.306 \\
\hline Time between courses & & & & & & & 0.639 \\
\hline$<30$ days & 140 & $(88.1)$ & 113 & $(86.9)$ & 27 & $(93.1)$ & \\
\hline $31-90$ days & 11 & $(6.9)$ & 10 & $(7.7)$ & 1 & $(3.5)$ & \\
\hline$>90$ days & 8 & $(5.0)$ & 7 & $(5.4)$ & 1 & $(3.5)$ & \\
\hline
\end{tabular}

\section{Discussion}

In our analysis, we found an overall CDI incidence of 0.979 per 10,000 residence days and a prevalence of $2.6 \%$. These risk and burden measures are consistent with rates of 0.8 to 14.1 cases $/ 10,000$ resident days or prevalence rates of $1.5-3.8 \%$ of nursing home admissions as was reported by Laffan et al. (2006). ${ }^{6}$ The highest incidence was found in residents with NNH-Acquired CDI. ${ }^{6}$

Residents who developed or had CDI were more likely to be treated with broad-spectrum antibiotics and have underlying conditions or illnesses that may have predisposed them to CDI. ${ }^{3,8,12}$ As has been noted by others, age $\geq 65$ years, female gender, cancer, corticosteroids, renal impairment, and solid organ transplant are patient characteristics associated with CDI. ${ }^{13-16}$ Increased exposure to antibiotics within the previous 3 months, use of nasogastric tubes, treatment with medications that affect the intestinal tract, and use of chemotherapeutic agents are also common risk factors of CDI. ${ }^{13}$ Recent hospitalization, close contact with infected individuals, and history of prior CDI are linked to elevated risk in infected individuals. ${ }^{13}$ Additionally, nursing home residents were shown to be more susceptible to CDI as a result of their generally diminished immune response, malnutrition, underlying comorbidities, cognitive deficits, incontinence, immobility, and other functional deficits. ${ }^{17,18}$ We were intrigued to find that coprescription of megestrol acetate was higher in residents with CDI than the control group. While the mechanism is not clear, residents typically receive megestrol acetate for either palliative treatment of terminal cancers or for cachexia, both of which can be associated with immunosuppression. Thus, megestrol's role in CDI may be correlative rather than causative. We note that, as has been documented in hospitalized patients, type 2 diabetes was correlated significantly with the presence of $\mathrm{NNH}$ Acquired CDI. 19,20

In our examination of $\mathrm{NNH}-$ and $\mathrm{NH}$-Acquired CDI, 2 distinctly different cohorts emerge. Residents transferred from other sites, predominantly acute care hospitals, were more likely to have renal disease, cancer, and underlying infections predisposing them to CDI. They were also more likely to be discharged to the hospital. In comparison, the chronic nursing home population who acquired CDI in the facility had more underlying functional and cognitive impairment, incontinence, and strokes. Despite the higher use of antibiotics and other medications associated with CDI in nursing home residents, these long-stay individuals represent a much smaller cadre of CDI and, although the severity of CDI illness could not be quantified, may have had a less significant form of the disease. The greatest risk of CDI in the nursing home appears to come from the transfer of infection from the hospital. CDI remains a problem in the long-term care setting, and as shown by others, importation from the acute care setting likely accounts for the majority of cases. ${ }^{21}$

In the absence of a means to assess the severity of CDI, we were unable to determine the appropriateness of the treatment choice as outlined in the SHEA/IDSA CDI treatment guidelines. ${ }^{11}$ However, given that initial and recurrence treatment with metronidazole is recommended for mild-to-moderate CDI, and oral vancomycin is recommended for initial and second-line treatment for those with severe disease, the findings in this nursing home population align with the expected treatment regimens suggested by SHEA/IDSA. ${ }^{11}$ 
Our results showed that retreatment for CDI is common and often occurs within 30 days of initial therapy. This suggests that either treatment failure or recurrence of the initial infection plays a large role in ongoing morbidity in these residents. The recurrence rate of CDI appears to increase with age and reaches rates as high as 38\% for patients older than aged 75 years treated initially with metronidazole; recurrence rates with vancomycin $(23 \%)$ remain unaffected by increased age. ${ }^{22}$ Louie et al. (2013) predicted a 17\% lower clinical cure rate, $17 \%$ greater recurrence, and 13\% lower sustained clinical response by advancing decade than in those younger than aged 40 years $(P<0.01$ each $) .^{23}$ Due to the tremendous burden CDI has on residents and on nursing home resources, strategies should be undertaken to identify and mitigate underlying risk factors in order to prevent the spread of CDI in this care environment. The Centers for Disease Control and Prevention (CDC) recommends contact precautions for the duration of illness, hand hygiene in compliance with CDC and the World Health Organization guidelines, specific regimens for cleaning and disinfection of affected equipment and care environments, laboratory-based alert systems, CDI surveillance, and education of staff as core strategies to reduce the occurrence of CDI. ${ }^{24}$ The Healthcare Infection Control Practices Advisory Committee is a federal advisory committee focused on the practice of infection control and strategies for surveillance, prevention, and control of health care-associated infections, which continues to advise CDC on practice improvements. ${ }^{25}$

\section{Limitations}

There are several limitations in our approach that must be taken into consideration prior to generalization of the results. The CDC surveillance definition of hospital-acquired CDI is similar but not identical to Mylotte's recommendations for surveillance of $C$. difficile in long-term care facilities, wherein infections within 30 days postdischarge are considered hospital acquired. ${ }^{9}$ The 30-day cutoff has recently been included in the National Healthcare Safety Network surveillance system for long-term care facilities using the LAB-ID event approach (http://www.cdc.gov/nhsn/PDFs/LTC/LTCF-LabID-EventProtocol_FINAL_8-24-12.pdf). It is possible that by using a 14-day cutoff, we may have underestimated the frequency of occurrence of NNH-Acquired CDI and consequently misclassified some of our NH-Acquired CDI residents as NNHAcquired. All in all, we were limited by the information that was available in the MDS.

As mentioned earlier, in the absence of a chart review, we were not able to determine the severity of the CDI and thus were unable to assess whether it was treated appropriately. This was not an objective of the study but is hypothesis-generating for the future.

Without knowing the exact index date of infection, we inferred an index date based on the first day that a prescription for oral vancomycin, oral metronidazole, or IV metronidazole was dispensed. It is possible that the true index date occurred before the first prescription fill. The resultant retreatment dates could underestimate the time between treatments.
Nevertheless, because of the overwhelming predominance of retreatment within 30 days of the initial treatment, it is reasonable to conclude that most retreatments resulted from recurrence or treatment failure rather than new infection. The analysis and interpretation of reasons for retreatment is limited by our inability to distinguish recurrence from new infections.

Due to the nature of the MDS source data, we had access to antibiotics and medications administered in the nursing home but did not have access to medications administered in the hospital. Thus, we were unable to conclude whether co-incident medication administration was causative or contributory to CDI in NNH-Acquired cases.

Use of the MDS has been criticized by some, noting that data discrepancies occur due to miscoding. Agreement rates for a number of data elements have been reportedly poor, but reliability has been shown to be adequate when using a weighted kappa statistic. ${ }^{26}$ While there are limitations to this caregiver-administered assessment instrument, it is a standardized tool that provides the "best available evidence" of resident status and outcomes in the nursing facility setting. When reviewed by others, MDS data have been reported to have moderate to moderately high validity and reliability. ${ }^{27} \mathrm{We}$ found that only $34 \%$ of residents with CDI reported diarrhea on their index MDS, which is much lower than what would be expected given that presence of unformed bowel movements is a major symptom of CDI. A probable explanation for this is that unlike the pharmacy claims data, which are continuous in nature, MDS data are discrete assessments. Thus, the timing of MDS administration relative to the onset of CDI infection and resultant symptoms, such as diarrhea, may not necessarily appear correlated. It was not possible to be certain whether the observed patient characteristics occurred prior to or after the index CDI infection.

MDS 3.0 data were not evaluated, since Section I.2.b [C. difficile], found on the MDS 2.0, was removed from MDS 3.0. Reliance on the MDS nurse to enter a specific text entry or ICD-9-CM code for CDI could lead to significant under-recognition of the true incidence of CDI.

\section{Conclusions}

Features of CDI, burden of illness, and treatment vary substantially between $\mathrm{NH}$-Acquired and $\mathrm{NNH}$-Acquired CDI. Retreatment of CDI is common and often occurs within 30 days of initial therapy. Those residents with NNH-Acquired CDI are more likely to be treated with vancomycin and be admitted to the hospital than those with NH-Acquired CDI. Residents with CDI carry a higher burden of illness compared with those without CDI and are more frequently admitted to the hospital. Unfortunately, we were not able to determine if the reason for discharge to the hospital was related specifically to CDI or to other underlying or acute conditions. It was also not possible to explore whether an existing but otherwise stable underlying condition was exacerbated by the CDI. Further study of this serious and growing infection in nursing facilities is warranted. 


\section{Authors}

BARBARA J. ZAROWITZ, PharmD, is Chief Clinical Officer and Vice President; CARRIE ALLEN, PharmD, is Associate Researcher, and TERRENCE O'SHEA, PharmD, is Senior Director, Consultant Pharmacist Measurement and Performance, Omnicare Senior Health Outcomes, Omnicare, Inc., Livonia, Michigan. MARCIE E. STRAUSS, MPH, was Associate Director, Health Economics and Outcomes Research, Cubist Pharmaceuticals, Lexington, Massachusetts.

AUTHOR CORRESPONDENCE: Barbara Zarowitz, PharmD, Omnicare Inc., 33510 Schoolcraft Rd., Livonia, MI 48150.

Tel.: 734.525.3308; Fax: 734.525.3344;

E-mail: barbara.zarowitz@omnicare.com.

\section{DISCLOSURES}

Zarowitz, Allen, and O'Shea are employees of Omnicare Senior Health Outcomes and receive grant funding from AbbVie, Amgen, Astellas, Forest, GlaxoSmithKline, Optimer/Cubist, and Sanofi. Zarowitz holds Omnicare stock. Strauss was an employee of Optimer/Cubist at the time this work was completed.

Study concept and design were primarily contributed by Strauss and O'Shea, with assistance from Allen and Zarowitz. Data collection was performed by Alexander Shif of Omnicare Senior Health Outcomes. Data were interpreted by Zarowitz, Allen, O'Shea, and Strauss. The manuscript was written and revised primarily by Zarowitz, along with Allen and O'Shea, with assistance from Strauss.

\section{ACKNOWLEDGMENTS}

We would like to thank Alexander Shif for his expertise and dedication to the data analytics for this project. Shif performed the database linking of Minimum Data Set and prescription claims data, de-identified the data, and subsequently populated the table shells. Shif is a Data Analyst for Omnicare Senior Health Outcomes, King of Prussia, Pennsylvania.

Additionally, we wish to thank Edward L. Peterson who provided an initial data analysis plan and sample size justification for the project and then performed the biostatistical analysis. Peterson is Division Head, Core Services, and Senior Biostatistician, Department of Public Health Sciences, Henry Ford Health System, Detroit, Michigan.

\section{REFERENCES}

1. Kelly CP, LaMont JT. Clostridium difficile-more difficult than ever. N Engl J Med. 2008;359(18):1932-40.

2. Pepin J, Alary ME, Valiquette L, et al. Increasing risk of relapse after treatment of Clostridium difficile colitis in Quebec, Canada. Clin Infect Dis. 2005;40(11):1591-97.

3. Kelly CP, Pothoulakis C, LaMont JT. Clostridium difficile colitis. N Engl J Med. 1994;330(4):257-62.

4. Maroo S, Lamont JT. Recurrent Clostridium difficile. Gastroenterol. 2006;130(4):1311-16.

5. Surawicz CM, Brandt LJ, Binion DG, et al. Guidelines for diagnosis, treatment and prevention of Clostridium difficile infections. Am J Gastroenterol. 2013;108(4):478-98.

6. Laffan AM, Bellatoni MF, Greenough WB 3rd, et al. Burden of Clostridium difficile-associated diarrhea in a long-term care facility. J Am Geriatr Soc. 2006;54(7):1068-73.

7. Centers for Disease Control and Prevention. Vital signs: preventing Clostridium difficile infections. MMWR Morb Mortal Wkly Rep. 2012;61(9):157-62.
8. Stevens V, Dumyati G, Fine LS, Fisher SG, van Wijngaarden E. Cumulative antibiotic exposures over time and the risk of Clostridium difficile infection. Clin Infect Dis. 2011;53(1):42-48.

9. Mylotte JM. Surveillance for Clostridium difficile-associated diarrhea in long-term care facilities: what you get is not what you see. Inf Cont Hosp Epidemiol. 2008;29(8):760-63.

10. Paquay L, De Lepeleire J, Schoenmakers B, Ylieff M, Fontaine O, Buntinx F. Comparison of the diagnostic accuracy of the Cognitive Performance Scale (Minimum Data Set) and the Mini-Mental State Exam for the detection of cognitive impairment in nursing home residents. Int $J$ Geriatr Psychiatry. 2007;22(4):286-93.

11. Cohen SH, Gerding DN, Johnson S, et al. Clinical practice guidelines for Clostridium difficile infection in adults: 2010 update by the Society for Healthcare Epidemiology of America (SHEA) and the Infectious Diseases Society of America (IDSA). Infect Control Hosp Epidemiol. 2010;31(5):431-55. 12. Bignardi GE. Risk factors for Clostridium difficile infection. J Hosp Infect. 1998;40(1):1-15.

13. McFarland LV. Renewed interest in a difficult disease: Clostridium difficile infections-epidemiology and current treatment strategies. Curr Opin Gastroenterol. 2009;25(1):24-35.

14. Loo VG, Bourgault AM, Poirier L, et al. Host and pathogen factors for Clostridium difficile infection and colonization. N Engl J Med. 2011; 365(18):1693-703.

15. Sheth H, Bernardini J, Burr R, et al. Clostridium difficile infections in outpatient dialysis cohort. Infect Control Hosp Epidemiol. 2010;31(1):89-91 16. Pant C, Anderson MP, O'Connor JA, Marshall CM, Deshpande A, Sferra TJ. Association of Clostridium difficile infection with outcomes of hospitalized solid organ transplant recipients: results from the 2009 Nationwide Inpatient Sample database. Transpl Infect Dis. 2012;14(5):540-47. 17. Zagaria MAE. Predisposition to infection in the elderly. US Pharm. 2011;36(8):28-31.

18. Gavazzi G, Krause KH. Ageing and infection. Lancet Infect Dis. 2002;2(11):659-66

19. Hassan SA, Rahman RA, Huda N, Wan Bebakar WM, Lee YY. Hospitalacquired Clostridium difficile infection among patients with type 2 diabetes mellitus in acute medical wards. J R Coll Physicians Edinb. 2013;43(2):103-07. 20. Shakov R, Salazar RS, Kagunye SK, Baddoura WJ, DeBari VA. Diabetes mellitus as a risk factor for recurrence of Clostridium difficile infection in the acute care hospital setting. Am J Infect Control. 2011;39(3):194-98.

21. Mylotte JM, Russell S, Sackett B, Vallone M, Antalek M. Surveillance for Clostridium difficile infection in nursing homes. J Am Geriatr Soc. 2013;61(1):122-25.

22. Johnson S, Gerding DN, Davidson TJ, et al. Efficacy and safety of oral vancomycin and metronidazole treatment for $C$. difficile associated diarrhea (CDAD): pooled results of two randomized clinical trials. Presented at: 2012 Annual Meeting of the Infectious Diseases Society of America; October 2012; San Diego, CA.

23. Louie TJ, Miller MA, Crook DW, et al. Effect of age on treatment outcomes in Clostridium difficile infection. J Am Geriat Soc. 2013;61(2):222-30. 24. Gould C, McDonald C. Clostridium difficile (CDI) infections toolkit. December 23, 2009. Centers for Disease Control and Prevention. Available at: http://www.cdc.gov/HAI/pdfs/toolkits/CDItoolkitwhite_clearance_edits. pdf. Accessed May 22, 2015.

25. Centers for Disease Control and Prevention. Healthcare Infection Control Practices Advisory Committee (HICPAC). Available at: http://www. cdc.gov/hicpac/about.html. Accessed May 22, 2015.

26. Mor V, Angelelli J, Jones R, Roy J, Moore T, Morris J. Inter-rater reliability of nursing home quality indicators in the U.S. BMC Health Services Res. 2003;3(1):20. Available at: http://www.ncbi.nlm.nih.gov/pmc/articles/ PMC280691/?tool=pubmed. Accessed May 22, 2015.

27. Shin JH, Scherer Y. Advantages and disadvantages of using MDS data in nursing research. J Gerontol Nurs. 2009;35(1):7-17. 


\section{Risk Factors, Clinical Characteristics, and Treatment Differences Between Residents With and Without Nursing Home- and Non-Nursing Home-Acquired Clostridium difficile Infection}

\section{APPENDIX Selected Treatment Index MDS Data Elements for Residents}

with CDI Compared with Control Group Without CDI ${ }^{a}$

\section{MDS Data Element or Medication}

Number of residents in MDS 2.0 Database with and without a diagnosis of CDI (frequency matched)

Demographics and Cognitive Status

Gender

Female

Male

Age range

\begin{tabular}{l}
\hline$<65$ \\
\hline $65-69$ \\
\hline $70-74$ \\
\hline $75-79$ \\
\hline $80-84$ \\
\hline $85-89$ \\
\hline$>89$ \\
\hline Mean age \pm SD \\
\hline Race/ethnicity
\end{tabular}

White, not of Hispanic origin

Black, not of Hispanic origin

Hispanic

Asian/Pacific Islander

N/A

American Indian/Alaskan native

Native Hawaiian/Pacific Islander

Degree of cognitive impairment

N/A

None-mild

Moderate

Severe

Physical Functioning/Functional Status

Bed mobility self-performance

N/A

Independent

Supervision

Limited assistance

Extensive assistance

Total dependence

Activity did not occur

Bed mobility support

N/A

No setup or physical help from staff

Setup help only

1 person physical assist

$2+$ person physical assist

ADL activity did not occur

Transfer self-performance

N/A

Independent

Supervision

Limited assistance

Extensive assistance

\begin{tabular}{c|c|c}
$\begin{array}{c}\text { With CDI Treatment } \\
\text { n (\%) }\end{array}$ & $\begin{array}{c}\text { Without CDI (Control) } \\
\mathbf{n}(\%)\end{array}$ & $\boldsymbol{P}_{\text {Value }}$ \\
\hline $2,048 \quad(100.0)$ & $2,048 \quad(100.0)$ & \\
\hline
\end{tabular}

1,257

791

273

177

196

289

385

416

312

$78.3 \pm 12.2$

(61.4)

(38.6)

(13.3)

(8.6)

(9.6)

(14.1)

(18.8)

(20.3)

(15.2)

\begin{tabular}{|r|r}
\hline 1,576 \\
\hline 246 \\
\hline 149 \\
\hline 48 \\
\hline 23 \\
\hline 0 \\
\hline 26 \\
\hline 1,243 \\
\hline 596 \\
\hline 178 \\
\hline
\end{tabular}

\begin{tabular}{r}
$(77.0)$ \\
$(12.0)$ \\
$(7.3)$ \\
$(2.3)$ \\
$(1.1)$ \\
$(0.3)$ \\
$(0.0)$ \\
\hline$(1.4)$ \\
\hline$(60.9)$ \\
\hline$(29.1)$ \\
$(8.7)$
\end{tabular}

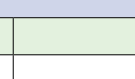

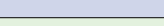
(8.7)

$2 \quad(0.1)$

88

60

312

1,281

305

0

(4.3)

(2.9)

(15.2)

(62.6)

(14.9)

(0.0)

$\begin{array}{ll}16 & (0.8) \\ 54 & (2.6)\end{array}$

$\begin{array}{rr}54 & (2.6) \\ 62 & (3.0) \\ 1,046 & (51.1)\end{array}$

1,046

0

(42.5)

(0.0)

(0.2)

(2.7)

(2.4)

(16.9)

(53.7)

347
1,100

\begin{tabular}{|l|l|}
\hline & \\
\hline & \\
\hline & \\
\hline & \\
\hline & \\
\hline & \\
\hline & \\
\hline
\end{tabular}

1,257

$791 \quad$ (38.6)

$(13.3)$
$(8.6)$
$(9.6)$
$(14.1)$
$(18.8)$
$(20.3)$
$(15.2)$
3.0

$78.1 \pm 13.0$

(61.4)

$\mid$

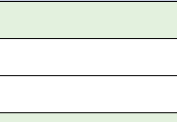

\begin{tabular}{|l|l}
\hline \\
\hline \\
\hline \\
\hline \\
\hline \\
\hline
\end{tabular}

\begin{tabular}{rrr|r}
\hline 1,576 & $(77.0)$ & \\
\hline 246 & $(12.0)$ & \\
\hline 149 & $(7.3)$ & \\
\hline 48 & $(2.3)$ & \\
\hline 23 & $(1.1)$ & \\
\hline 6 & $(0.3)$ & \\
\hline 0 & $(0.0)$ & $<0.01$ \\
\hline & & \\
\hline & $(1.2)$ & \\
\hline 687 & $(33.4)$ & \\
\hline 243 & $(11.9)$ & \\
\hline \multicolumn{3}{|c}{} \\
\end{tabular}

$<0.01$

$\begin{array}{rr}3 & (0.2) \\ 281 & (13.7)\end{array}$

$113 \quad$ (5.5)

$370 \quad(18.1)$

$1,029 \quad(50.2)$

$252 \quad(12.3)$

$0 \quad(0.0)$

$71 \quad(3.5)$

$200 \quad$ (9.8)

$131 \quad(6.4)$

$1,048 \quad(51.2)$

$598 \quad(29.2)$

$0 \quad(0.0)$

$4 \quad(0.2)$

$\begin{array}{rr}218 & (10.6) \\ 132 & (6.5)\end{array}$

$390 \quad(19.0)$

$899 \quad(43.9)$

\begin{tabular}{l}
$<0.01$ \\
\hline$<$ \\
\hline$<0.01$ \\
\hline$<$ \\
\hline$<0.01$ \\
\hline
\end{tabular}




\section{Risk Factors, Clinical Characteristics, and Treatment Differences Between Residents With and Without Nursing Home- and Non-Nursing Home-Acquired Clostridium difficile Infection}

\section{APPENDIX Selected Treatment Index MDS Data Elements for Residents with}

CDI Compared with Control Group Without CDI ${ }^{a}$ (continued)

MDS Data Element or Medication

Total dependence

Activity did not occur

Transfer support

N/A

No setup or physical help from staff

Setup help only

1 person physical assist

$2+$ person physical assist

ADL activity did not occur

Walk in room

N/A

Independent

Supervision

Limited assistance

Extensive assistance

Total dependence

Activity did not occur

ADL score mean \pm SD

Continence

Urinary continence

\section{N/A \\ Continent}

Occasionally incontinent

Frequently incontinent

Incontinent

Bowel incontinence

N/A

Continent

Occasionally incontinent

Frequently incontinent

Incontinent

Bowel elimination pattern

Diarrhea

Miscellaneous

Proton-pump inhibitors

Statins

Nutritional Status

Nutritional approaches

Parenteral/IV

Feeding tube

Parenteral or enteral intake ${ }^{c}$

Proportion of calories received through parenteral or tube feedings

N/A

$25 \%$ or less

$26 \%$ to $50 \%$

$51 \%$ or more

Average fluid intake by IV or tube feeding

N/A

$500 \mathrm{cc}$ or less

$501 \mathrm{cc}$ or more
With CDI Treatment

n (\%)

$446 \quad(21.8)$

48

(2.3)

$17 \quad(0.8)$

$31 \quad(1.5)$

$37 \quad(1.8)$

867

1,048

48

(42.3)

(51.2)

(2.3)

2

2
71

(0.1)

(3.5)

(4.0)

(19.0)

390

272

15

1,216

$14.1 \pm 3.2$

(13.3)

(0.7)

(59.4)

\section{$14.1 \pm 3.2$}

$\begin{array}{rr}3 & (0.2) \\ 709 & (34.6) \\ 326 & (15.9) \\ 417 & (20.4) \\ 593 & (29.1)\end{array}$

2

514

313

390

829

700

(0.1)

(25.1)

(15.3)

(19.0)

(40.5)

Without CDI (Control) n (\%)

$\begin{array}{rr}378 & (18.5) \\ 27 & (1.3)\end{array}$

71

71
117

117

871

25

(3.5)

(8.2)

(5.7)

(42.5)

(38.9)

$25 \quad(1.2)$

3

(0.2)

257

150

395

(12.6)

$(7.3)$

(19.3)

(12.0)

$\begin{array}{rr}246 & (12.0) \\ 11 & (0.5) \\ 986 & (48.1) \\ 12.5 \pm 4.2\end{array}$

$P$ Value $^{\mathrm{b}}$

$12.5 \pm 4.2$

\begin{tabular}{l|l}
$<0.01$ \\
\hline
\end{tabular}

(34.2)

2
767

407

311

564

$(0.1)$

(37.5)

(19.7)

(15.2)

(27.5)

1 938

938

(0.1)

(45.8)

(13.1)

$207 \quad(10.1)$

634

(31.0)

77

(3.8)

$<0.01$

1,275

884

(62.3)

(43.2)

1,057
825

(51.6)

(40.3)

$<0.01$

0.06

$306 \quad(14.9)$

160

(7.8)

66

146

(3.2)

$(7.1)$

0.41

\begin{tabular}{|c|c|c|c|c|}
\hline & & & & 0.49 \\
\hline 10 & $(0.5)$ & 11 & $(0.5)$ & \\
\hline 113 & $(5.5)$ & 58 & $(2.8)$ & \\
\hline 18 & $(0.9)$ & 7 & $(0.3)$ & \\
\hline 4 & $(0.2)$ & 4 & $(0.2)$ & \\
\hline 293 & (14.3) & 126 & $(6.2)$ & \\
\hline & & & & 0.12 \\
\hline 9 & $(0.4)$ & 11 & $(0.5)$ & \\
\hline 72 & (3.5) & 43 & (2.1) & \\
\hline 357 & (17.4) & 152 & (7.4) & \\
\hline
\end{tabular}


Risk Factors, Clinical Characteristics, and Treatment Differences Between Residents With and

Without Nursing Home- and Non-Nursing Home-Acquired Clostridium difficile Infection

APPENDIX Selected Treatment Index MDS Data Elements for Residents with

CDI Compared with Control Group Without CDI ${ }^{\text {(continued) }}$

\begin{tabular}{|c|c|c|c|c|c|}
\hline MDS Data Element or Medication & With C & eatment & Without & (Control) & $P$ Value ${ }^{b}$ \\
\hline \multicolumn{6}{|l|}{ Special Treatments and Procedures } \\
\hline Dialysis & 127 & $(6.2)$ & 63 & $(3.1)$ & $<0.01$ \\
\hline Chemotherapy & 13 & $(0.6)$ & 10 & $(0.5)$ & 0.53 \\
\hline \multicolumn{6}{|l|}{ Discharge Status ${ }^{d}$} \\
\hline Hospital & 600 & $(29.3)$ & 301 & $(14.7)$ & 0.001 \\
\hline Deceased & 110 & $(5.4)$ & 40 & $(2.0)$ & 0.297 \\
\hline \multicolumn{6}{|c|}{$\begin{array}{l}\text { andex MDS defined as first MDS assessment during the quarter when the index MDS for the treatment group was completed. } \\
\text { bP values indicate category comparison to residents with CDI in MDS } 2.0 \text { time frame. } \\
\text { 'Residents with discharged assessments within } 30 \text { days after Index MDS. } \\
\text { dOnly residents with parenteral/IV or feeding tube counted. } \\
\text { ADL=activities of daily living; } c c=\text { cubic centimeters; CDI=Clostridium difficile infection; IV=intravenous; MDS= Minimum Data Set 2.0; N/A=not available; SD=standard } \\
\text { deviation. }\end{array}$} \\
\hline
\end{tabular}

